\title{
CdSe Sensitized Thin Aqueous Films: Probing the Potential Distribution Inside Multilayer Assemblies ${ }^{\dagger}$
}

\author{
Mohamad Hojeij, Nicolas Eugster, Bin Su, and Hubert H. Girault* \\ Laboratoire d'Electrochimie Physique et Analytique, Ecole Polytechnique Fédérale de Lausanne, Station 6, \\ CH-1015 Lausanne, Switzerland
}

Received April 28, 2006. In Final Form: July 19, 2006

\begin{abstract}
Ultrathin polypeptide multilayer films are assembled by the sequential electrostatic adsorption of monolayers of poly-L-lysine and poly-L-glutamic acid onto carboxylic acid terminated alkanethiol-modified gold surfaces. The polypeptide multilayer films are hydrophilic, can incorporate electroactive species such as ferri/ferrocyanide, and are stable when immersed in organic solvents such as 1,2-dichloroethane. Cadmium selenide quantum dots stabilized by negatively charged citrate groups are electrostatically attached to the multilayer film assembly in order to act as photoactive species. Photocurrent responses originating from the CdSe sensitized ultrathin multilayer film are investigated as functions of the applied potential, the thickness of the film and the presence of quenchers in the organic phase. A theoretical model is proposed in order to analyze the kinetics of the photoinduced electron-transfer reactions and to probe the potential distribution within the film.
\end{abstract}

\section{Introduction}

In the past decade, considerable effort has been devoted to the fabrication of novel ultrathin films featuring various functionalities. ${ }^{1-3}$ An interesting approach to the fabrication of multilayer ultrathin films is the sequential layer-by-layer deposition of oppositely charged polyelectrolytes. This method was first proposed by Decher and co-workers ${ }^{4-7}$ by extending the pioneering work of Iler et al. ${ }^{8}$ The thickness of the films obtained with this technique can be easily controlled at the nanometer level by varying the number of adsorbed polyelectrolyte layers. ${ }^{9-12}$ Layer-by-layer deposition can be extended to a wide variety of other interesting charged materials, such as dendrimers, ${ }^{13}$ light-emitting polymers, ${ }^{14}$ and metallic ${ }^{15,16}$ or semiconducting materials. ${ }^{17}$ The possibility to use colloidal nanoparticles as building blocks in these films has recently been demonstrated. ${ }^{16-20}$ Semiconducting nanoparticles composed of elements from the periodic groups II-VI (CdSe, CdS, CdTe, ZnSe, ...),

\footnotetext{
$\dagger$ Part of the Electrochemistry special issue.

* To whom correspondence should be addressed. Tel: +4121 6933151 . Fax: +41 21693 3667. E-mail: hubert.girault@epfl.ch.

(1) Decher, G.; Schlenoff, J. B. Multilayer assembly films: Sequential Assembly of Nanocomposite Materials; Wiley-VCH: Weinheim, Germany, 2003.

(2) Tripathy, S. K.; Kumar, J.; Nalwa, H. S. Handbook of polyelectrolytes and their applications; American Scientific Publishers: Steveson Ranch, CA, 2002, Vols. 1 and 3.

(3) Hammond, P. T. Curr. Opin. Colloid Interface Sci. 2000, 4, 430.

(4) Decher, G.; Hong, J. D. Ber. Bunsen-Ges.-Phys. Chem. Chem. Phys. 1991 95,1430 .

(5) Decher, G. Science 1997, 277, 1232

(6) Decher, G.; Hong, J. D.; Schmitt, J. Thin Solid Films 1992, 210, 831.

(7) Decher, G.; Eckle, M.; Schmitt, J.; Struth, B. Curr. Opin. Colloid Interface

Sci. 1998, 3, 32 .

(8) Iler, R. K. J. Colloid Interface Sci. 1966, 21, 569.

(9) Lvov, Y.; Haas, H.; Decher, G.; Mohwald, H.; Kalachev, A. J. Phys. Chem. 1993, 97, 12835

(10) Lvov, Y.; Ariga, K.; Onda, M.; Ichinose, I.; Kunitake, T. Langmuir 1997, 13,6195 .

(11) Bouldemais, F.; Schwinté, P.; Gergely, C.; Voegel, J. C.; Schaaf, P. Langmuir 2002, 18, 4523.

(12) Cheng, Y. F.; Corn, R. M. J. Phys. Chem. B 1999, 103, 8726.

(13) Kabanov, V. a.; Zezin, A. B.; Rogacheva, V. B.; Gulyaeva, Z. G.; Zansochova, M. F.; Joosten, J. G. H.; Brackman, J. Macromolecules 1999, 32 1904.

(14) Onoda, M.; Yoshino, K. J. Appl. Phys. 1995, 78, 4456.

(15) Pastoriza-Santos, I.; Koktysh, D. S.; Mamedov, A. A.; Giersig, M.; Kotov, N. A.; Liz-Marzan, L. M. Langmuir 2000, 16, 2731.

(16) Hao, E. C.; Lian, T. Q. Chem Mater. 2000, 12, 3392

(17) Kotov, N. a.; Dekany, I.; Fendler, J. H. J. Phys. Chem. 1995, 99, 13065

(18) Cassagneau, T.; Mallouk, T. E.; Fendler, J. H. J. Am. Chem. Soc. 1998 120,7848
}

also called quantum dots (QDs), present unique optical, electrical, and magnetic properties, which can be tuned by varying the nanoparticle size. ${ }^{21-26}$ The incorporation of QDs inside multilayer films therefore provides a novel approach to the fabrication of photoactive modified electrodes.

In a recent publication, we showed that various porphyrin species could be self-assembled onto multilayer films from solution. ${ }^{27}$ Photocurrent responses similar to those obtained at the liquid|liquid interface ${ }^{28-30}$ were observed in the presence of the electron acceptors $\mathrm{C}_{60}$ and tetracyanoquinodimethane (TCNQ) in the organic phase. The incident photon to current conversion efficiency for this type of cells is of about $0.02 \%$ at $0 \mathrm{~V}$, which corresponds to a quantum efficiency of $0.15 \% .^{27,31}$ These modest performances reflect the low charge carrier efficiencies of the immobilized redox ions. On the other hand, 3-D architectures of metallic particles could be obtained by alternatively depositing layers of polycations and citrate-protected gold nanoparticles. ${ }^{19,31,32}$ Particle densities of the order of $30 \%$ could be obtained without significant aggregation of the particles. A strong orbital coupling was observed between the particles and the gold surface, allowing the transport of electrons inside the film.

In the present paper, we extend this approach by depositing semiconducting nanoparticles onto the multilayer film. Our longterm goal is to develop a device which allows efficient light energy conversion, while retaining the relatively low cost and

(19) Kakkassery, J. J.; Abid, J. P.; Carrara, M.; Fermin, D. J. Faraday Discuss. 2004, 125, 157.

(20) Carrara, M.; Kakkassery, J. J.; Abid, J. P.; Fermin, D. J. Chem. Phys Chem. 2004, 5, 571 .

(21) Alivisatos, A. P. Science 1996, 271, 933

(22) Alivisatos, A. P. J. Phys. Chem. 1996, 100, 13226.

(23) Nirmal, M.; Brus, L. Acc. Chem. Res. 1999, 32, 407.

(24) Murphy, C. J.; Coffer, J. L. Appl. Spectrosc. 2002, 56, 16A

(25) Cottingham, K. Anal. Chem. 2005, 77, 354A.

(26) Zhang, J. Z. J. Phys. Chem. B 2000, 104, 7239

(27) Kakkassery, J. J.; Fermin, D. J.; Girault, H. H. Chem. Commun. 2002, 1240.

(28) Eugster, N.; Fermin, D. J.; Girault, H. H. J. Phys. Chem. B 2002, 106 , 3428.

(29) Eugster, N.; Fermin, D. J.; Girault, H. H. J. Am. Chem. Soc. 2003, 125, 4862.

(30) Fermin, D. J.; Duong, H. D.; Ding, Z. F.; Brevet, P. F.; Girault, H. H. J. Am. Chem. Soc. 1999, 121, 10203 .

(31) Kakkassery, J. J. Organisation and electrochemical reactivity at molecular interfaces. Ph.D. Thesis; EPFL: Switzerland, 2004.

(32) Chirea, M.; Garcia-Morales, V.; Manzanares, J. A.; Pereira, C.; Gulaboski, R.; Silva, F. J. Phys. Chem. B 2005, 109, 21808. 
ease of preparation of the multilayer assembly. Citrate-stabilized QDs provide a promising approach to this problem, as their photoreactivity appears unaffected by their adsorption onto the film (see section 2.4). Furthermore, these nanoparticles can be used not only as sensitizers but also as structural elements of the electrostatic assembly. Preliminary results from our group reveal that 3-D architectures containing several layers of QDs exhibit excellent light energy conversion. However, several questions concerning this system remain to be addressed in order to optimize the efficiency. The purpose of the present paper is to employ CdSe particles as probes to analyze the potential distribution inside polyelectrolyte multilayer films. We shall demonstrate that most of the potential drop between the gold substrate and the electrolyte solution is concentrated within the first five polyelectrolyte layers.

We have recently shown that the excitation of mercaptosuccinic acid stabilized CdSe nanoparticles assembled at the water|1,2dichloroethane (DCE) interface results in efficient heterogeneous electron transfer to acceptors located in the adjacent phase. ${ }^{33}$ This electron-transfer manifests itself as photocurrent responses under potentiostatic conditions. The photoinduced process was analyzed in terms of a kinetic model based on our previous work at porphyrin sensitized liquid|liquid boudaries. ${ }^{34,35} \mathrm{We}$ shall demonstrate that the dynamics of photoinduced electron-transfer reactions in CdSe sensitized polyelectrolyte assembly can be analyzed by a similar kinetic modeling. Photocurrent transients are recorded at various conditions of applied potential and film thickness. The rates of electron transfer across the film, as well as that of charge recombination and coupled reactions, are extracted from the photocurrent data using a relatively simple kinetic model.

\section{Experimental Section}

2.1. Chemicals. All reagents used were of analytical grade. PolyL-lysine (pLys; $M_{\mathrm{w}}=30300$ ) and poly-L-glutamic acid (pGlu; $M_{\mathrm{w}}$ $=40000$ ) were purchased from Sigma. 11-Mercaptoundecanoic acid (MUA) and ferrocene were purchased from Aldrich. Potassium ferricyanide $\left(\mathrm{K}_{3}\left[\mathrm{Fe}(\mathrm{CN})_{6}\right]\right)$, potassium ferrocyanide $\left(\mathrm{K}_{4}\left[\mathrm{Fe}(\mathrm{CN})_{6}\right]\right)$, disodium hydrogen phosphate $\left(\mathrm{Na}_{2} \mathrm{HPO}_{4} \cdot 2 \mathrm{H}_{2} \mathrm{O}\right)$, sodium dihydrogen phosphate $\left(\mathrm{NaH}_{2} \mathrm{PO}_{4} \cdot \mathrm{H}_{2} \mathrm{O}\right)$, and bis(triphenylphosphoranylidene) ammonium chloride (BTPPAC1) are Fluka products. Lithium tetrakis(pentafluorophenyl) borate (LiTPFB) was purchased from Boulder Scientific. 1,2-Dichloroethane (DCE, Aldrich) and ethanol (Aldrich) were used as solvents. The supporting electrolyte in the organic phase was bis-(triphenylphosphoranylidene) ammoniumtetrakis (pentafluorophenyl) borate (BTPPATPFB), which was prepared as previously reported. ${ }^{36}$ All aqueous solutions were prepared with purified water from a Milli-Q 185 system. Finally, citrate-stabilized $\mathrm{CdSe}$ quantum dots were prepared according to a reported procedure ${ }^{37}$ In a typical synthesis, $0.05 \mathrm{~g}$ of trisodium citrate and $2 \mathrm{~mL}$ of cadmium perchlorate $0.04 \mathrm{~mol} \mathrm{dm}^{-3}$ were added to $45 \mathrm{~mL}$ of water, and the $\mathrm{pH}$ was then adjusted to 9.0 with sodium hydroxide. The solution was bubbled with nitrogen for $10 \mathrm{~min}$, before the addition of $2 \mathrm{~mL}$ of $N, N$-dimethylselenourea $0.01 \mathrm{~mol} \mathrm{dm}^{-3}$. The mixture was heated in a microwave oven at $900 \mathrm{~W}$ for $50 \mathrm{~s}$. The resulting solution has a transparent orange color and contains CdSe QDs electrostatically stabilized by the anions in the solution.

2.2. Preparation of Gold Electrodes. Glass slides were treated with Piranha solution and cleaned by sequential sonication in ethanol,

(33) Su, B.; Fermin, D. J.; Abid, J. P.; Eugster, N.; Girault, H. H. J. Electroanal. Chem. 2005, 583, 241 .

(34) Samec, Z.; Eugster, N.; Fermin, D. J.; Girault, H. H. J. Electroanal. Chem. 2005, 577, 323 .

(35) Su, B.; Eugster, N.; Girault, H. H. J. Am. Chem. Soc. 2005, 127, 10760

(36) Fermin, D. J.; Duong, H. D.; Ding, Z. F.; Brevet, P. F.; Girault, H. H. Phys. Chem. Chem. Phys. 1999, 1, 1461.

(37) Rogach, A. L.; Nagesha, D.; Ostrander, J. W.; Giersig, M.; Kotov, N. A Chem. Mater. 2000, 12, 2676.

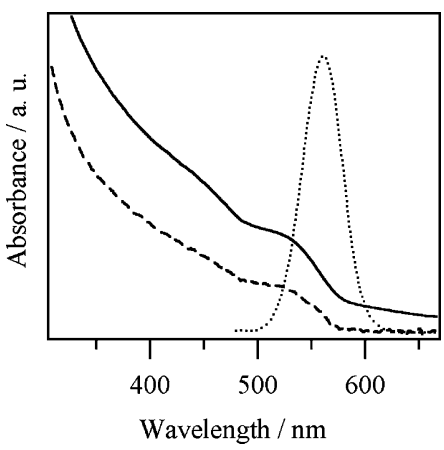

Figure 1. Absorption (solid line) and emission (dotted line) spectra of an aqueous colloidal CdSe QDs solution. The emission was recorded after excitation at $442 \mathrm{~nm}$. Absorption spectrum of the CdSe QDs adsorbed onto 3 layers of pLys and pGlu (dashed line). The deposition time was $1 \mathrm{~h}$.

acetone and purified water followed by drying under a stream of Argon. The gold films (100 nm) were prepared in an Edwards Auto 306 evaporator operating at a pressure less than $5 \times 10^{-6} \mathrm{mbar}$. The film growth was initiated by the thermal evaporation of a $1 \mathrm{~nm}$ thick chromium $\left(99.99 \%\right.$, Balzers) layer on the glass slide at $<0.1 \mathrm{~nm} \mathrm{~s}^{-1}$ to enhance the adhesion of the Au layer. Gold (99.99\%, Balzers) was subsequently evaporated at $<0.1 \mathrm{~nm} \mathrm{~s}^{-1}$ up to $5 \mathrm{~nm}$, before increasing the deposition rate to $0.2-0.3 \mathrm{~nm} / \mathrm{s}$ for the deposition of a $100 \mathrm{~nm}$ layer.

2.3. Film Preparation. The gold substrates were initially modified with a self-assembled monolayer of thiol by immersion into an ethanolic solution of $10^{-3} \mathrm{~mol} \mathrm{dm}{ }^{-3}$ mercaptoundecanoic acid (MUA) for $12 \mathrm{~h}$. Loosely bound thiol molecules were removed from the surface by rinsing successively with ethanol and water before drying the electrode under an argon stream. The preparation of polyelectrolyte multilayer films is based on the method described in detail by Corn and co-workers. ${ }^{12,38}$ Solutions of pLys $\left(1 \mathrm{~g} \mathrm{dm}^{-3}\right)$ and pGlu $\left(1 \mathrm{~g} \mathrm{dm}^{-3}\right)$ were prepared in a phosphate buffer at $\mathrm{pH}$ 8.4. The multilayer polyelectrolyte films were prepared by the sequential dipping of the MUA-modified gold electrode into alternating solutions of pLys and pGlu for $15 \mathrm{~min}$, starting and ending with a pLys layer. The deposition time of 15 min was assigned from Kelvin probe measurements. ${ }^{31}$ Between each dipping, the surface was rinsed with Millipore water and blown dry under an argon stream. Incorporation of ferri/ferrocyanide ions into the film was carried out afterward by dipping the multilayer film in a solution of ferrocyanide and ferricyanide $10^{-3} \mathrm{~mol} \mathrm{dm}^{-3}$ in phosphate buffer ( $\mathrm{pH}$ 5.6) for $15 \mathrm{~min}$, followed by the rinsing and drying procedure described above.

Polyelectrolyte multilayer films ending with a layer of pLys feature a positively charged surface appropriate for the adsorption of citrate stabilized QDs. CdSe QDs were electrostatically attached to the pLys modified electrode surface by introducing the film into the colloidal CdSe solution for $1 \mathrm{~h}$, followed by rinsing and drying.

2.4. Characterization of the CdSe QDs. Transmission electron microscopy (TEM) was used to analyze the size of the prepared nanoparticles. The TEM images reveal that the CdSe QDs have a quasi-spherical shape with an average size around $2.5 \mathrm{~nm}$. The absorption and emission spectra of the aqueous colloidal CdSe solution are displayed in Figure 1. The absorption spectrum exhibits a shoulder in a range between 490 and $580 \mathrm{~nm}$ where the $1 \mathrm{~s}-1 \mathrm{~s}$ electronic transitions occur. ${ }^{37,39,40}$ The emission spectrum of the CdSe shows a bell shape in the spectral range of 500-600 nm, with a maximum at $561 \mathrm{~nm}$ and a fwhm around $48 \mathrm{~nm}$.

The electrostatic adsorption of CdSe particles onto the polyelectrolyte multilayer assembly can then be monitored by UV-vis absorption spectroscopy. The absorption spectrum of CdSe QDs

(38) Cheng, Y. F.; Murtomaki, L.; Corn, R. M. J. Electroanal. Chem. 2000 483,88 .

(39) Schooss, D.; Mews, A.; Eychmuller, A.; Weller, H. Phys. Rev. B 1994 $49,17072$.

(40) Javier, A.; Yun, C. S.; Sorena, J.; Strouse, G. F. J. Phys. Chem. B 2003 $107,435$. 


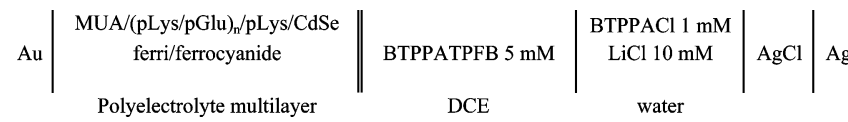

Figure 2. Representation of the electrochemical cell employed in photocurrent measurements.

deposited onto three layers of pLys/pGlu is shown in dashed line in Figure 1. The multilayer assembly in the absence of CdSe was used as a reference in order to isolate the spectral features associated with the adsorbed particles. The optical properties of the CdSe QDs appear unaffected by the adsorption. No obvious aggregation of the nanoparticles is observed on the film surface. No significant increase in the absorption was observed for longer deposition times, indicating that the adsorption is complete within $1 \mathrm{~h}$.

2.5. Instruments. The electrochemical cell used in all measurements is a homemade three-electrode cell with a cylindrical glass vessel. The multilayer modified gold slide functions as the working electrode, with a Pt wire counter electrode and an $\mathrm{Ag} / \mathrm{AgCl}$ reference electrode. The junction between the reference electrode and the organic electrolyte was established by an aqueous solution containing $10^{-2} \mathrm{~mol} \mathrm{dm}^{-3} \mathrm{LiCl}$ and $10^{-3} \mathrm{~mol} \mathrm{dm}^{-3} \mathrm{BTPPACl}$ as indicated in Figure 2. The 1,2-dichloroethane phase contained $5 \times 10^{-3} \mathrm{~mol}$ $\mathrm{dm}^{-3}$ of BTPPATPFB as supporting electrolyte. All potentials reported refer to the $\mathrm{Ag} / \mathrm{AgCl}$ electrode. The potential difference between the electrode and the organic solution was controlled with a homemade potentiostat. The absorption and emission measurements were performed on an Ocean Optics CHEM2000 UV-vis spectrophotometer and a Perkin-Elmer LS50B luminescence spectrometer, respectively. TEM measurements were performed on a Philips CM20 microscope with an accelerating voltage of $200 \mathrm{kV}$ and a point resolution of $0.28 \mathrm{~nm}$.

2.6. Photocurrent Measurements. Photocurrent measurements were performed under potentiostatic conditions using the illumination provided by the $442 \mathrm{~nm}$ beam line of a $\mathrm{CW} \mathrm{He}-\mathrm{Cd}$ laser (Omnichrome Series 74). Neutral density filters were used for controlling the photon flux. The reference electrode was kept in the dark to avoid any photopotential interferences. Transient photocurrent measurements were conducted employing a mechanical shutter. The photon flux was measured using a calibrated power meter (Gentec). The illumination and current recording were synchronized by means of homemade Labview programs.

The photoelectrochemical measurements were carried out in 1,2dichloroethane in order to prevent the diffusion of aqueous ions from the film. Indeed, when the film is immersed in aqueous solution, the large difference in volumes makes it difficult to maintain the concentration of free ions inside the film. Furthermore, the use of an organic electrolyte solution immiscible with the aqueous environment of the film ensures that the redox species placed in the solution, such as TCNQ, do not enter the film in substantial quantity. It is however difficult to predict what effect the contact of an organic liquid will have on the arrangement of the hydrophilic polyelectrolyte chains and charged nanoparticles. Finally, it should be mentioned that the solutions were left in contact with air during photocurrent measurements. The system does hence contain molecular oxygen, which can eventually act as an electron acceptor.

\section{Results and Discussion}

As mentioned in the Introduction, the photoactivity of dyesensitized multilayer films was described in a previous publication. ${ }^{27}$ The presence of zinc porphyrins, together with incorporated redox centers, allows electron transfer between the film and the gold surface upon illumination. In the present paper, the CdSe nanoparticles take the role of photoactive species at the surface of the film. Illumination of the modified electrode leads to the formation of electron-hole pairs in the CdSe particles. The transport of charges through the film is mediated by the presence of ferri/ferrocyanide. Depending on the potential profile across the film, electron transport occurs either from the gold surface
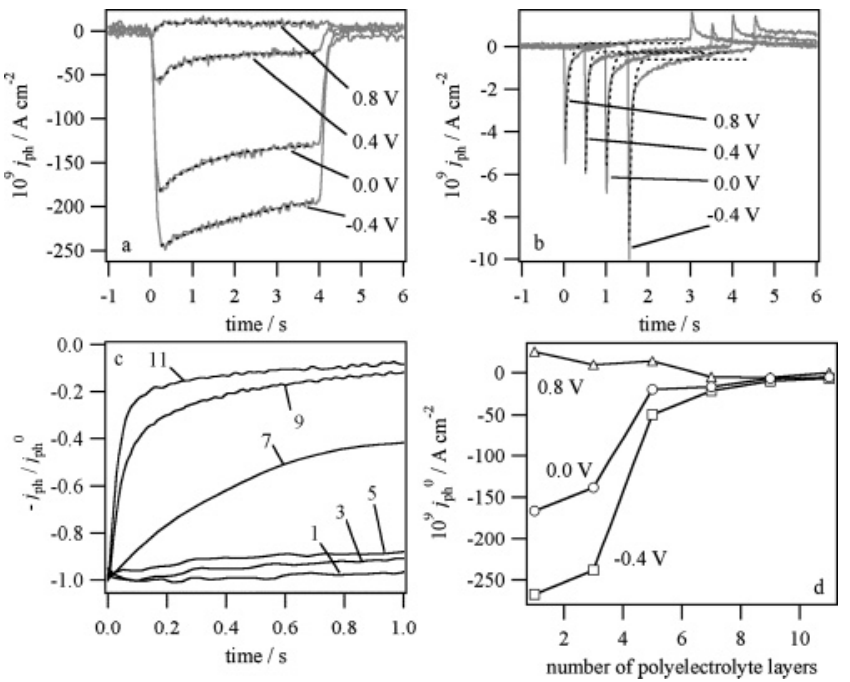

Figure 3. Photocurrent transient responses of CdSe sensitized multilayer film at various applied potentials. The films were composed of 3 (a) and 9 (b) layers of pLys and pGlu (i.e., $n$ in Figure 2 is equal to 1 and 4, respectively). The dashed lines are fittings employing eq 13. Normalized photocurrent transients for various film thicknesses at $-0.4 \mathrm{~V}(\mathrm{c})$. The number of pLys and pGlu layers is indicated next to each curve. Initial photocurrent as a function of the number of pLys and pGlu layers (d) at -0.4 (squares), 0.0 (circles), and 0.8 $\mathrm{V}$ (triangles). The initial photocurrent is the maximum photocurrent value observed directly upon illumination.

to the CdSe particles (photoreduction) or from the particles to gold (photooxidation).

Based on unpublished data from our group, the electron transport inside the film is believed to proceed mostly via electron hopping from redox site to redox site, rather than by diffusion of the ferri/ferrocyanide ions. Indeed, analysis of electron transfer reactions between the gold surface and redox species in the multilayer assembly using cyclic voltammetry and electrochemical impedance suggests that the electron transfer inside the film is faster than the diffusion of ionic species. We expect these results to be part of a future publication. The distribution of the electrical potential from the gold electrode to the organic phase through the aqueous film is the key parameter controlling the apparent rate of electron transfer between the CdSe particles and the electrode, and hence the amplitude of the photocurrent responses. Although the overall potential drop between the gold surface and the electrolyte solution can be easily controlled experimentally, we have little information on the potential distribution inside the multilayer assembly. Kelvin probe measurements have revealed that the addition of alternate pLys and pGlu layers on the electrode results in an oscillatory behavior of the contact potential difference. ${ }^{20}$ These results reflect changes in the multilayer surface potential. This behavior could be reflected in the potential distribution, depending on the level of structure of the film. On the other hand, as the distance between the gold surface and the nanoparticles is increased by adding more pLys and pGlu layers the potential distribution should tend to that of two polarizable interfaces in series, namely the gold|film and the film|organic phase interface.

The photoinduced electron transfer between the particles and the gold surface manifests itself as photocurrent responses. Figure 3 , panels a and $b$, shows the photocurrent transients at various potentials obtained for different film thicknesses. No faradaic response was observed prior to the illumination. Furthermore, no photocurrents were observed in the absence of CdSe QDs on the surface of the film. Upon illumination, the photocurrent rises sharply to a maximum initial value $j_{\mathrm{ph}}^{0}$. In the following seconds 


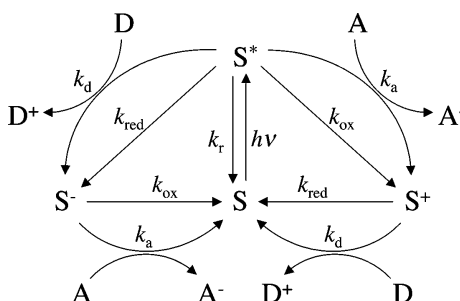

Figure 4. Schematic representation of the photoinduced electrontransfer reactions. (S) corresponds to a CdSe particle (sensitizer). The species (A) and (D) correspond to electron accepting and donating species in the organic phase.

the photocurrent decreases slowly due to recombination currents (see section 3.1). When the illumination is stopped, the photocurrent decreases back to zero, and in the case of thick films a current overshoot is observed. This overshoot is associated with back charge transfer reactions occurring when the film contains negatively charged particles. A positive current corresponding to the photooxidation of the CdSe particles can be observed at high potential differences when the film is composed of 7 or less polyelectrolyte layers. This is apparent in Figure 3a at $0.8 \mathrm{~V}$.

The photocurrent decay observed after the initial photocurrent $j_{\text {ph }}^{0}$ is reached is much more pronounced in the case of thick films. This can be clearly seen in Figure 3c, which displays the first seconds of the photocurrent decay normalized by the initial photocurrent. Two regimes can be distinguished: in the case of films composed of less than 7 pLys and pGlu layers, the photocurrent decay is relatively slow, whereas it is much sharper with films featuring more than 7 layers. Photocurrents obtained in the case of 7 polyelectrolyte layers appear as intermediates between these two situations.

Not only the shape but also the photocurrent magnitude is strongly affected by changes in the potential difference and the number of layers. Photocurrent responses increase when the potential is tuned to more negative values, suggesting that the rate of electron transfer from the gold substrate to the QDs is increased. On the other hand, the photocurrent markedly decreases when more polyelectrolyte layers are added. This phenomenon is clearly illustrated by Figure 3d, which displays the initial photocurrent as a function of the number of layers at various potentials. The effect of the potential difference is investigated in section 3.2, whereas the dependence of the photocurrent on the film thickness is discussed in section 3.3.

3.1. Theoretical Description of the Photocurrent Responses. To rationalize the photocurrent data presented in Figure 3, we shall develop a kinetic model based on previous investigations of dye-sensitized liquid|liquid interfaces. The overall photoinduced process can be described by the scheme in Figure 4. A similar modelisation of the photoprocesses involving semiconducting nanoparticles attached to electrode surfaces can be found in the work of Hickey et al. ${ }^{41-43}$ The photocurrent response is deconvoluted in a series of competing reactions. The initial step consists of the photoexcitation of the CdSe particles, denoted S in Figure 4.

$$
\mathrm{S} \stackrel{h v}{\longrightarrow} \mathrm{S}^{*}
$$

The excited particle, $\mathrm{S}^{*}$, can either relax to the ground state by fluorescence or nonradiative pathways, or participate in electron-

(41) Hickey, S. G.; Riley, D. J. J. Phys. Chem. B 1999, 103, 4599

(42) Hickey, S. G.; Riley, D. J. Electrochim. Acta 2000, 45, 3277.

(43) Hickey, S. G.; Riley, D. J.; Tull, E. J. J. Phys. Chem. B 2000, 104, 7623. transfer reactions with the surrounding iron centers and ultimately the gold surface.

$$
\begin{gathered}
\mathrm{S}^{*} \stackrel{k_{\mathrm{r}}}{\longrightarrow} \mathrm{S} \\
\mathrm{S}^{*} \stackrel{k_{\mathrm{ox}}}{\longrightarrow} \mathrm{S}^{+}+\mathrm{e}^{-}(\mathrm{Au}) \\
\mathrm{S}^{*}+\mathrm{e}^{-}(\mathrm{Au}) \stackrel{k_{\mathrm{red}}}{\longrightarrow} \mathrm{S}^{-}
\end{gathered}
$$

This mechanism is based on several assumptions: First, an average rate constant $k_{\mathrm{r}}$ is assigned to the decay of the excited state, without consideration of trap states and additional decay pathways. Second, the transfer of electrons across the film is simplified as two first-order kinetic processes with rate constants $k_{\text {ox }}$ and $k_{\text {red. }}$. These rate constants are associated with the oxidation and reduction of the particles, respectively. Obviously, the mechanism through which electrons are shuttled between the gold substrate and the particles at the surface of the film is much more complex and involves multiple redox reactions between iron centers, as well as the diffusion of redox species inside the film. These charge-transfer reactions are influenced by the local film structure and potential profile. The rate constants $k_{\mathrm{ox}}$ and $k_{\text {red }}$ should be considered as the reciprocals of the average time of electron transport through the films.

The CdSe nanoparticles carrying extra charges can be recycled through different mechanisms: back charge transfer or quenching via redox species present in the film or in the organic electrolyte solution. For instance, in the case of reduced CdSe particles, the reactions can be considered to be

$$
\begin{aligned}
& \mathrm{S}^{-} \stackrel{k_{\mathrm{ox}}}{\longrightarrow} \mathrm{S}+\mathrm{e}^{-}(\mathrm{Au}) \\
& \mathrm{S}^{-}+\mathrm{A} \stackrel{k_{\mathrm{a}}}{\longrightarrow} \mathrm{S}+\mathrm{A}^{-}
\end{aligned}
$$

where $\mathrm{A}$ is an electron acceptor located either in the film or in the organic phase, such as TCNQ (see section 3.4). The rate constant associated with dark oxidation of $\mathrm{S}^{-}$(5) can be considered, as a first approximation, to be equal to the rate constant for the oxidation of excited CdSe. Similar reactions can be considered in the case of the oxidized CdSe particles

$$
\begin{aligned}
& \mathrm{e}^{-}(\mathrm{Au})+\mathrm{S}^{+} \stackrel{k_{\mathrm{red}}}{\longrightarrow} \mathrm{S} \\
& \mathrm{S}^{+}+\mathrm{D} \stackrel{k_{\mathrm{d}}}{\longrightarrow} \mathrm{S}+\mathrm{D}^{+}
\end{aligned}
$$

where $\mathrm{D}$ is an electron donor in the system and not in the organic electrolyte solution. Obviously, the quenching of electrons in the conduction band or holes in the valence band of CdSe can occur from the excited particles $S^{*}$ as well. The following reactions should hence be considered:

$$
\begin{aligned}
& \mathrm{S}^{*}+\mathrm{A} \stackrel{k_{\mathrm{a}}}{\longrightarrow} \mathrm{S}^{+}+\mathrm{A}^{-} \\
& \mathrm{S}^{*}+\mathrm{D} \stackrel{k_{\mathrm{d}}}{\longrightarrow} \mathrm{S}^{-}+\mathrm{D}^{+}
\end{aligned}
$$

These reactions are in competition with the electron-transfer reactions ( 3 and 4 ). The magnitude and shape of the photocurrent response will be controlled by the kinetics of each individual step. As apparent in Figure 3, the rate constants $k_{\mathrm{ox}}$ and $k_{\text {red }}$ are influenced by the applied potential, as well as the number of layers.

It should be mentioned that the measured photocurrent corresponds to an electron transfer reaction at the gold|film interface, whereas the concomitant charge transfer reactions at 
the film|organic phase interface can either be an interfacial electron transfer between CdSe particles and a donor or acceptor in the organic phase or an ion transfer reaction across the interface to maintain the electroneutrality of the multilayer film. In the present model, we do not take into account the ion transfer reactions that may or may not take place as these do not influence directly the oxidation states of the nanoparticles. We shall therefore consider the electron flux across the gold|film junction in order to estimate the photocurrent without having to take into account ion transfer processes

$$
j_{\mathrm{ph}}=F\left(\left(k_{\mathrm{ox}}-k_{\mathrm{red}}\right) \Gamma_{\mathrm{S}^{*}}+k_{\mathrm{ox}} \Gamma_{\mathrm{S}^{-}}-k_{\mathrm{red}} \Gamma_{\mathrm{S}^{+}}\right)
$$

where $\Gamma_{i}$ denotes the surface concentration of the sensitizer in its excited, oxidized, and reduced forms, respectively. Assuming that a preequilibrium takes place between $S$ and $S^{*}$, the concentration of excited particles can be described as

$$
\Gamma_{\mathrm{S}^{*}}=\frac{I_{0} \sigma \Gamma_{\mathrm{S}}^{0}}{k_{\mathrm{r}}}
$$

where $I_{0}$ is the photon flux, $\sigma$ is the capture cross-section of the particles, and $\Gamma_{\mathrm{S}}^{0}$ is the total surface concentration of particles adsorbed on the film. Of course, eq 12 is valid only in the case where the number of excited nanoparticles remains small (i.e., $\left.k_{\mathrm{r}} \gg I_{0} \sigma\right)$. Using a Laplace transform on the concentration changes of $\mathrm{S}^{+}$and $\mathrm{S}^{-}$, the following expression can be obtained for the photocurrent: ${ }^{29,34}$

$$
j_{\mathrm{ph}}=\Phi\left(\alpha-\beta \mathrm{e}^{-\left(k_{\mathrm{ox}}+k_{\mathrm{a}}\right) t}+\gamma \mathrm{e}^{-\left(k_{\mathrm{red}}+k_{\mathrm{d}}\right) t}\right)
$$

with

$$
\begin{gathered}
\alpha=\frac{\left(k_{\mathrm{ox}} k_{\mathrm{d}}-k_{\text {red }} k_{\mathrm{a}}\right)\left(k_{\text {ox }}+k_{\text {red }}+k_{\mathrm{a}}+k_{\mathrm{d}}\right)}{\left(k_{\mathrm{ox}}+k_{\mathrm{a}}\right)\left(k_{\text {red }}+k_{\mathrm{d}}\right)} \\
\beta=\frac{k_{\text {ox }}\left(k_{\text {red }}+k_{\mathrm{d}}\right)}{\left(k_{\text {ox }}+k_{\mathrm{a}}\right)} \\
\gamma=\frac{k_{\text {red }}\left(k_{\text {ox }}+k_{\mathrm{a}}\right)}{\left(k_{\text {red }}+k_{\mathrm{d}}\right)}
\end{gathered}
$$

and

$$
\Phi=F \frac{I_{0} \sigma \Gamma_{\mathrm{S}}^{0}}{k_{\mathrm{r}}}
$$

The first term in the photocurrent equation describes the steadystate current. The second and third terms are associated with the current decays at short illumination times. The initial photocurrent value observed directly upon illumination of the film $(t=0)$ is simply described by

$$
j_{\mathrm{ph}}^{0}=\Phi\left(k_{\mathrm{ox}}-k_{\mathrm{red}}\right)
$$

The influence of the parameters $k_{\mathrm{ox}}, k_{\mathrm{red}}, k_{\mathrm{d}}$, and $k_{\mathrm{a}}$ are examined in Figure 5. The simulations in Figure 5 were obtained using $\Phi$ $=10^{-11} \mathrm{C} \mathrm{cm}^{-2}, k_{\mathrm{ox}}=2 \mathrm{~s}^{-1}, k_{\mathrm{red}}=10^{4} \mathrm{~s}^{-1}, k_{\mathrm{d}}=2 \times 10^{3} \mathrm{~s}^{-1}$, and $k_{\mathrm{a}}=1 \mathrm{~s}^{-1}$ unless specified otherwise in the figures. This set of values generates theoretical curves, which are close to what is observed experimentally. Under conditions where the photocurrent is negative, we have $k_{\text {red }} \gg k_{\text {ox }}$, so that the photocurrent is mainly controlled by $k_{\text {red }}$. In this case, the rate constant $k_{\mathrm{ox}}$ determines the sharpness of the photocurrent decay observed in
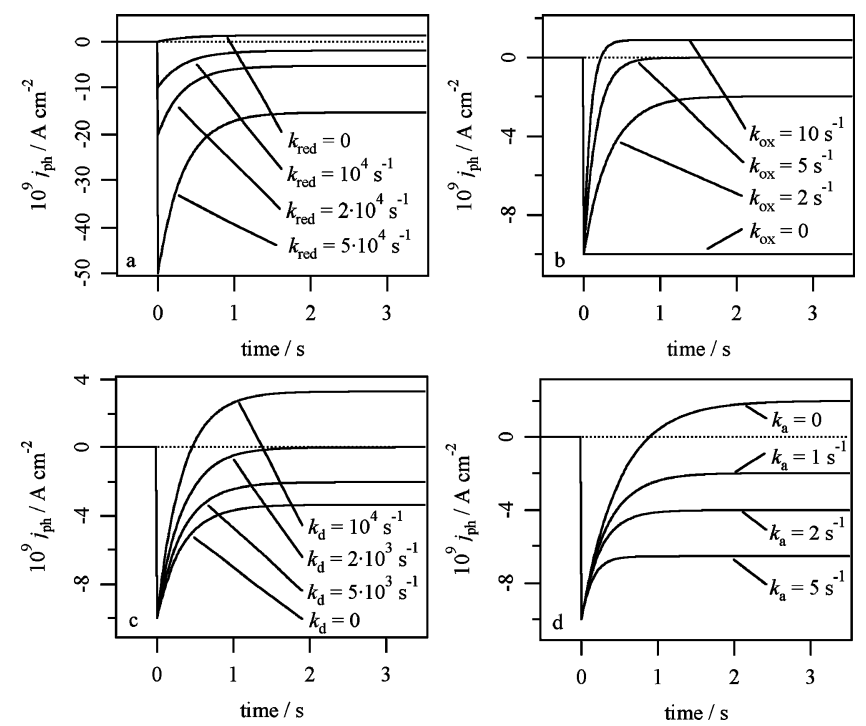

Figure 5. Simulation of the photocurrent responses using eq 13. The various parameters were taken as $\Phi=10^{-11} \mathrm{C} \mathrm{cm}^{-2}, k_{\mathrm{ox}}=2$ $\mathrm{s}^{-1}, k_{\text {red }}=10^{4} \mathrm{~s}^{-1}, k_{\mathrm{d}}=2 \times 10^{3} \mathrm{~s}^{-1}$, and $k_{\mathrm{a}}=1 \mathrm{~s}^{-1}$ unless specified otherwise in the figure.

the millisecond time scale, as it corresponds to a recombination pathway. By making a qualitative comparison between the photocurrent responses in Figure 3 and the simulations in Figure 5 , panels a and $b$, it can be seen that an increase in the potential acts to decrease $k_{\text {red }}$ and increase $k_{\text {ox }}$. At very positive potentials in the case of films with less than 7 layers, $k_{\mathrm{ox}}$ becomes greater than $k_{\text {red }}$, and we change from a reductive to an oxidative process. This situation can be exemplified by comparing the simulation curve corresponding to $k_{\text {red }}=0$ in Figure 5 a with the experimental curve at $0.8 \mathrm{~V}$ in Figure $3 \mathrm{a}$. As observed in Figure $5 \mathrm{c}$, a relatively large rate constant $k_{\mathrm{d}}$ is required in order to observe photocurrents that change sign over time. This situation occurs in the case of 7 layers at intermediate potentials. Finally, the rate constant of electron transfer to an acceptor in the organic phase controls the value of the steady-state value of a reduction photocurrent. This effect will be investigated in section 3.4.

The large number of parameters in eq 13 prevents an accurate fitting of the photocurrent data. To extract information on $k_{\mathrm{ox}}$ and $k_{\text {red }}$ from the data in Figure 3, we need to limit the number of adjustable parameters. The values of $k_{\mathrm{d}}, k_{\mathrm{a}}$, and $\Phi$ are not expected to vary much with the potential difference. A general comparison between the simulation curves in Figure 5 and the photocurrent data reveals that $k_{\mathrm{d}}=2000 \mathrm{~s}^{-1}$ can be employed to describe most transients. This value is close to the $2800 \mathrm{~s}^{-1}$ obtained experimentally by Hickey et al. for the capture of photogenerated holes in surface bound CdS nanoparticles. ${ }^{41}$ This reductive quenching may arise from a reaction with the surrounding polypeptides or solvent molecules. In this case, the reaction can be considered homogeneous, and the rate constant $k_{\mathrm{d}}$ is not expected to vary with the applied potential. Furthermore, we shall consider that changes in the thickness of the film do not introduce changes in the number of adsorbed particles, so that the parameter $\Phi$ is constant. The photon flux $I_{0}$ was measured as $2.5 \times 10^{16} \mathrm{~cm}^{-2} \mathrm{~s}^{-1}$ using a calibrated photodiode. The photon capture cross section $\sigma$ of the CdSe nanoparticles at $442 \mathrm{~nm}$ can be estimated as $10^{-15} \mathrm{~cm}^{2}$. ${ }^{44,45}$ The absorption spectra of CdSe sensitized films provide a rough estimate of the particle coverage

(44) Palik, E. D. Handbook of Optical Constants; Academic Press: Boston, 1991; Vol. 2

(45) Van De Hulst, H. C. Light Scattering by Small Particles; Courier Dover Publications: New York, 1981. 

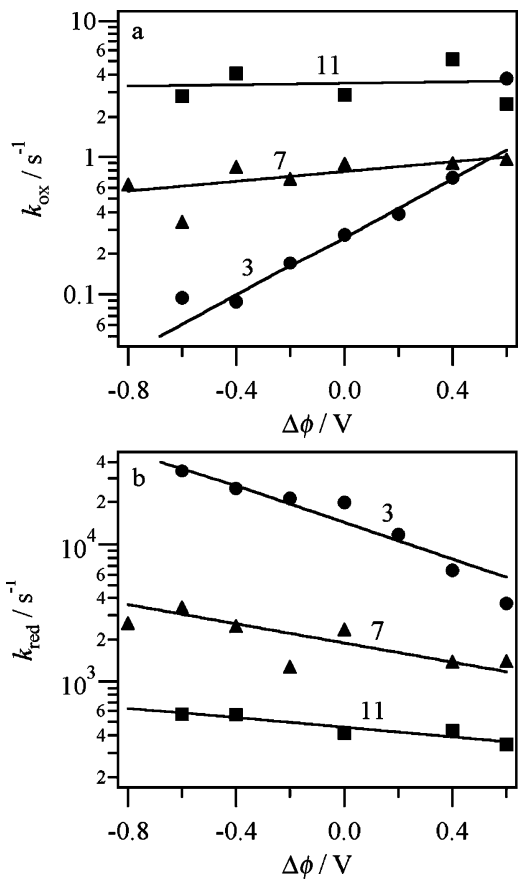

Figure 6. Rate constants $k_{\text {ox }}$ (a) and $k_{\text {red }}$ (b) as functions of the potential for 3 (circles), 7 (triangles), and 11 layers (squares) of pLys and pGlu. The rate constants were extracted from the photocurrent data using eq 13 with $\Phi=10^{-11} \mathrm{C} \mathrm{cm}^{-2}, k_{\mathrm{d}}=2 \times$ $10^{3} \mathrm{~s}^{-1}$, and $k_{\mathrm{a}}$ between 1 and $5 \mathrm{~s}^{-1}$. The black lines are guides for the eye.

as $\Gamma_{\mathrm{S}}^{0}=4 \times 10^{-11} \mathrm{~mol} \mathrm{~cm}^{-2}$. Finally, the rate constant $k_{\mathrm{r}}$ was determined as $10^{7} \mathrm{~s}^{-1}$ from fluorescence lifetime measurements (unpublished data from our group). Thus, the parameter $\Phi$ can be estimated as $10^{-11} \mathrm{C} \mathrm{cm}^{-2}$. This value was found to be consistent with the photocurrent data and was used in all fittings.

Under these assumptions in the case of a photoreduction, the initial photocurrent is mainly determined by $k_{\text {red }}$, and the slow photocurrent decay is associated with the recombination rate constant $k_{\mathrm{ox}}$ and the heterogeneous rate of electron transfer $k_{\mathrm{a}}$. In the absence of electron acceptor in the aqueous phase, the value of $k_{\mathrm{a}}$ remains in the range of $1-5 \mathrm{~s}^{-1}$. This residual quenching of the electrons in the CdSe conduction band may result from a reaction with oxygen. Indeed, solutions were left in contact with air, and the system can be considered as saturated with $\mathrm{O}_{2}$. Fittings of the photocurrent responses employing eq 13 are shown as dashed lines in Figure 3, panels a and b. It can be observed that, although some parameters are taken as constants, eq 13 provides an adequate description of the photocurrent transients, particularly in the case of low numbers of polyelectrolyte layers. When the films are composed of a large number of layers, such as in Figure 3b, the experimental transients deviate from the exponential behavior predicted by our model. This may be explained by the fact that several electron transfer reactions are needed to transport charges between the particles and the gold electrode. The photooxidation process, which is associated with the photocurrent decay, may not be adequately described by a single rate constant $k_{\mathrm{ox}}$ in the case of thick films.

3.2. Dependence of the Photocurrent Responses on the Applied Potential. It is clear from Figure 3 that the photocurrent strongly depends on the applied potential difference between the gold surface and the organic electrolyte solution. The influence of the potential suggests that a significant portion of the potential distribution takes place between the gold and the CdSe particles. Similar results were obtained in the case of porphyrin-sensitized multilayer assemblies. ${ }^{27}$ Such behavior is also consistent with
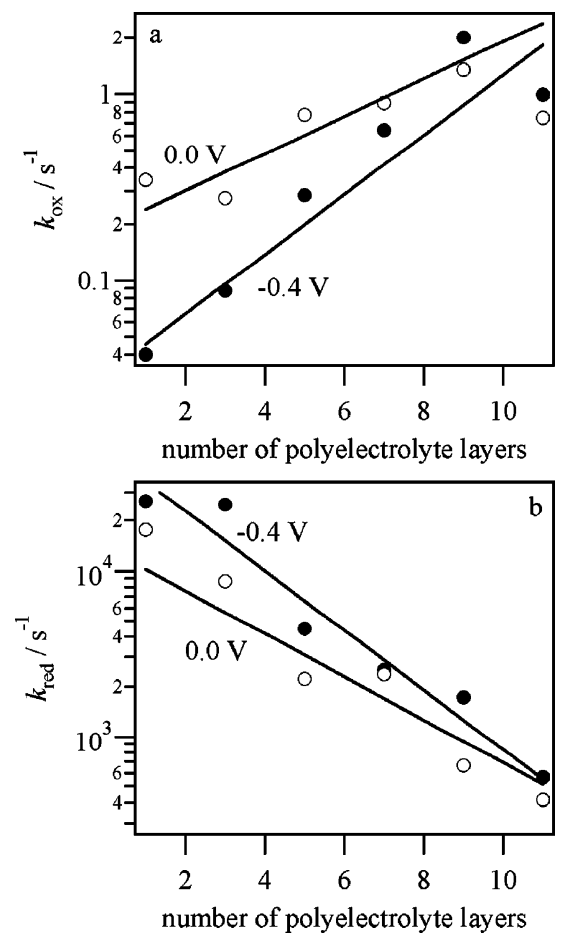

Figure 7. Rate constants $k_{\text {ox }}$ (a) and $k_{\text {red }}$ (b) as functions of the number of polyelectrolyte layers at -0.4 (black circles) and $0 \mathrm{~V}$ (white circles). The rate constants were extracted from the photocurrent data using eq 13 with $\Phi=10^{-11} \mathrm{C} \mathrm{cm}^{-2}, k_{\mathrm{d}}=2 \times 10^{3} \mathrm{~s}^{-1}$, and $k_{\mathrm{a}}$ between 1 and $5 \mathrm{~s}^{-1}$. The black lines are guides for the eye.

the work of Hickey et al., ${ }^{41-43}$ who employed CdS particles directly attached to thiol-modified gold electrodes.

The photocurrent transients in Figure 3 are negative for most of the applied potential differences, suggesting that $k_{\text {red }} \gg k_{\text {ox }}$. The more negative the potential is, the larger the reductive photocurrent response becomes. At potentials above $0.6 \mathrm{~V}$ in the case of films composed of less than 7 polyelectrolyte layers, the electron transfer from CdSe to gold becomes dominant, and positive oxidation photocurrents are observed (see Figure 3d). Figure 6 displays the rate constants $k_{\text {ox }}$ and $k_{\text {red }}$ as functions of the potential difference for various film thicknesses. An increase in the potential difference results in an increase of $k_{\mathrm{ox}}$ while $k_{\text {red }}$ decreases. The energy levels of excited electrons and holes in the CdSe particles are shifted with respect to the Fermi level of the gold substrate. Accordingly, the driving force associated with $k_{\mathrm{ox}}$ increases, whereas that of $k_{\text {red }}$ decreases.

3.3. Effect of the Film Thickness on the Photocurrent Responses. As discussed previously, the photocurrent data seem to show two distinct kinetic regimes depending on the thickness of the film. The system goes from a situation as in Hickey et al. ${ }^{41-43}$ where particles are separated from the gold surface by a dielectric layer of MUA to a 20-30 nm wide film which functions as a separate phase with relatively low conduction. ${ }^{11,12,27,31}$ The kinetics of electron transfer between gold and the CdSe QDs are expected to change accordingly.

From the data in Figure 3, it is evident that the initial photocurrent decreases when the number of layers is increased. This suggests that the CdSe particles are indeed positioned at the film|electrolyte boundary and do not freely penetrate inside the multilayer assembly. The photocurrent decay is also more pronounced when the film is thicker. These phenomena are reflected by the evolution of the rate constants of photooxidation and photoreduction. Figure 7 displays the rate constants $k_{\mathrm{ox}}$ and $k_{\text {red }}$ as extracted from the photocurrent data. Although the decrease in $k_{\text {red }}$ with the number of layers can be explained by the larger 


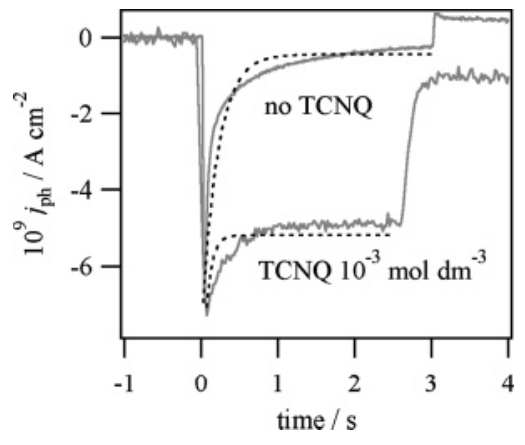

Figure 8. Photocurrent responses obtained at $-0.4 \mathrm{~V}$ with 11 layers of pLys and pGlu in the absence and in the presence of TCNQ $10^{-3}$ mol $\mathrm{dm}^{-3}$ in the DCE solution. The dashed lines are simulations using eq 13 with the parameters $\Phi=10^{-11} \mathrm{C} \mathrm{cm}^{-2}, k_{\mathrm{ox}}=1.25 \mathrm{~s}^{-1}$, $k_{\text {red }}=780 \mathrm{~s}^{-1}, k_{\mathrm{d}}=2 \times 10^{3} \mathrm{~s}^{-1}$, and $k_{\mathrm{a}}=3.5$ or $12 \mathrm{~s}^{-1}$.

distance separating the particles from the gold surface, the increase in $k_{\mathrm{ox}}$ is more surprising. A possible explanation is that, in the case of thick films, the potential difference is spread over a larger distance, so that the electron hopping from iron center to iron center is less disfavored.

As can be seen in Figure 6, the effect of the potential difference on the rate constants $k_{\mathrm{ox}}$ and $k_{\text {red }}$ is less marked in the case of thicker films. In the presence of 11 pLys and pGlu layers, the electron transfer rate constants are practically potential independent. These results suggest that the potential difference extends only over 5-7 polyelectrolyte layers, which corresponds to a distance of about $15 \mathrm{~nm}$. Indeed, theoretical modeling of the potential distribution in similar multilayer systems by GarciaMorales et al. indicate that most of the potential drop occurs within the first few layers. ${ }^{46}$ The adsorption of additional pLys and pGlu layers creates a portion of film with a weak potential distribution. The driving force for electron hopping within this region is low, which limits the overall rate of electron transfer across the film.

3.4. Photocurrent in the Presence of TCNQ in the Organic Solution. Photocurrent responses obtained in the absence and in the presence of TCNQ $5 \times 10^{-3} \mathrm{~mol} \mathrm{dm}^{-3}$ in DCE at $-0.4 \mathrm{~V}$ with a film composed of 11 layers of pLys and pGlu are displayed in Figure 8. A clear feature of these results is the shift of the steady-state reductive photocurrents to more negative values. The TCNQ molecules in the DCE phase can accept electrons from the $\mathrm{CdSe}$ conduction band, thereby decreasing the probability of back electron transfer from CdSe to gold. In terms of the scheme in Figure 4, this corresponds to an increase of the rate constant $k_{\mathrm{a}}$. The dashed curves in Figure 8 were obtained from

(46) Garcia-Morales, V.; Silva, T. H.; Moura, C.; Manzanares, J. A.; Silva, F. J. Electroanal. Chem. 2004, 569, 111. eq 13 using the parameters $\Phi=10^{-11} \mathrm{C} \mathrm{cm}^{-2}, k_{\mathrm{ox}}=1.25 \mathrm{~s}^{-1}$, $k_{\text {red }}=780 \mathrm{~s}^{-1}$, and $k_{\mathrm{d}}=2 \times 10^{3} \mathrm{~s}^{-1}$. The rate constant $k_{\mathrm{a}}$ of electron transfer to an acceptor was varied from $3.5 \mathrm{~s}^{-1}$ in the absence of TCNQ to $12 \mathrm{~s}^{-1}$ in the presence of the quencher. This rather small change suggests that TCNQ does not efficiently accept electrons from the CdSe particles. This may arise from the fact that the polyelectrolyte film forms a hydrophilic environment restricting the approach of TCNQ toward the particles.

\section{Conclusion}

Ultrathin films composed of alternate layers of pLys and pGlu were prepared on mercaptoundecanoic acid modified gold electrodes. The electroactivity of these films was ensured by the incorporation of ferri/ferrocyanide ions. Citrate-stabilized CdSe quantum dots were then electrostatically adsorbed on the film, conferring photoactive properties to the assembly. The optical properties of the particles were not changed upon deposition onto the multilayer assemblies suggesting that no aggregation phenomena occurrs.

Photocurrent responses originating from the photoreduction of the adsorbed CdSe nanoparticles were investigated in 1,2dichloroethane. The dynamics of photoinduced processes and coupled reactions are evaluated on the basis of a detailed kinetic model. The rate constants of electron transfer between the gold substrate and the particles was found to depend on the potential difference between gold and the organic electrolyte solution, as well as on the thickness of the multilayer assembly. At very positive potentials, the direction of the electron flux could be reversed from a reductive to an oxidative process. These results demonstrate that the potential distribution occurs through the two polarizable interfaces in series.

The distance between the gold surface and the CdSe particles was varied by changing the number of polyelectrolyte layers. We observed that the kinetics of CdSe photoreduction are decreased when the thickness of the multilayer assembly is increased. Furthermore, the dependence of the photocurrent responses on the potential difference becomes markedly weaker in the case of films composed of more than 7 pLys and pGlu layers. These data suggest that the electric field at the gold|film interface spreads over 5-7 polyelectrolyte layers, which corresponds to a distance of about $15 \mathrm{~nm}$.

Acknowledgment. We are grateful to Dr. Guillaume Meriguet and Dr. Shuxin Tan for the fruitful discussions. The technical assistance by Valérie Devaud is also acknowledged. This work was supported by the Swiss National Science Foundation (Project 20-67050.01).

LA0611561 